\title{
Femtosecond and Attosecond Electron-Transfer Dynamics in PCPDTBT:PCBM Bulk Heterojunctions
}

\author{
F. O. L. Johansson, ${ }^{\dagger}$ M. Ivanović, ${ }^{\ddagger}$ S. Svanström, ${ }^{\dagger}$ U. B. Cappel, ${ }^{\dagger} \S^{\circledR}$ H. Peisert, ${ }^{\ddagger}$ T. Chassé, \\ and A. Lindblad ${ }^{*}+$ (i)
}
${ }^{\dagger}$ Department of Physics and Astronomy, Molecular and Condensed Matter Physics, Uppsala University, Box 516, SE-75120 Uppsala, Sweden

${ }^{\ddagger}$ Institut für Physikalische und Theoretische Chemie, Universität Tübingen, Auf der Morgenstelle 18, D-72076 Tübingen, Germany

Supporting Information

ABSTRACT: Charge separation efficiency is a crucial parameter for photovoltaic devices-polymers consisting of alternating electron-rich and electron-deficient parts can achieve high such efficiencies, for instance, together with a fullerene electron acceptor. This offers a viable path toward solar cells with organic bulk heterojunctions. Here, we measured the charge-transfer times in the femtosecond and attosecond regimes via the decay of sulfur 1s X-ray coreexcited states (with the core-hole clock method) in blends of a
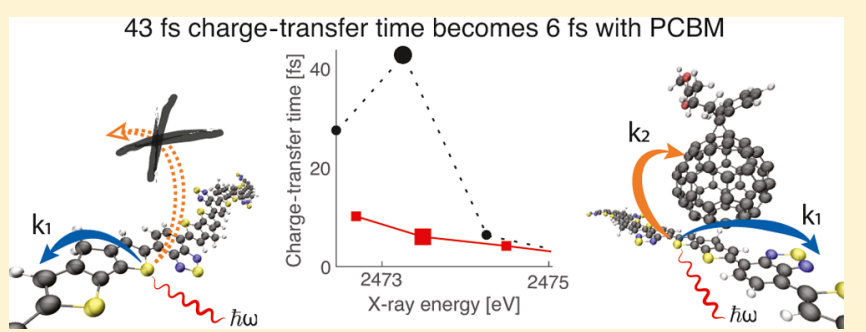

low-band gap polymer \{PCPDTBT [poly[2,6-(4,4-bis(2-ethylhexyl)-4H-cyclopenta[2,1-b;3,4- $\left.b^{\prime}\right]$ dithiophene)-alt-4,7-(2,1,3benzothiadiazole) $]]\}$ consisting of a cyclopentadithiophene electron-rich part and a benzothiadiazole electron-deficient part. The constituting parts of the bulk heterojunction were varied by adding the fullerene derivative PCBM $\left([6,6]-\right.$ phenyl-C $_{61}$-butyric acid methyl ester) (weight ratio of polymer/PCBM as 1:0, 1:1, 1:2, and 1:3). For low-energy excitations, the charge-transfer time varies to the largest extent for the thiophene donor part. The charge-transfer time in the $1: 2$ blend is reduced by $86 \%$ compared to that of pristine PCPDTBT. At higher energy excitations, the charge-transfer time does not vary with the chemical environment, as this regime is dominated by intramolecular conduction that yields ultrafast charge-transfer times for all blends, approaching 170 as. We thus demonstrate that the core-hole clock method applied to a series with changing composition can give information about local electron dynamics (with chemical specificity) at interfaces between the constituting parts-the crucial part of a bulk heterojunction where the initial charge separation occurs.

\section{INTRODUCTION}

Organic photovoltaic (OPV) devices offer a route toward cheap solar energy harnessing and are therefore an active research field where the interplay between chemistry and physics is the key to push device performance and longevity. ${ }^{1-3}$ As in other branches of (opto)electronics, polymer-based OPVs are attractive alternatives to silicon technology because of their relatively low cost, flexibility, coloration, and semitransparency. Furthermore, they are nontoxic and recyclable. These properties make them ideal candidates for alternative markets with ample opportunities, such as large projects and utilities and building-integrated photovoltaics. Projections to medium- and large-scale production scenarios predict cost targets up to $5 €$ cents per watt peak for OPV modules. ${ }^{4}$

The heart of the OPV cell is a bulk heterojunction $(\mathrm{BHJ})$ (Figure 1), which separates excitons into electron-hole pairs. An approach to improve the efficiency of donor-acceptorbased BHJ OPV cells is the use of low-band gap polymers with alternating electron-deficient and electron-rich subunits as donor materials. The lower optical band gap results in an improved efficiency because of an increased absorption in the visible and near-infrared regions of the solar spectrum. The polymer PCPDTBT (poly[2,6-(4,4-bis(2-ethylhexyl)-4H- cyclopenta[2,1-b;3,4- $\left.b^{\prime}\right]$ dithiophene)-alt-4,7-(2,1,3-benzothiadiazole)], structure in Figure 1$)^{6-8}$ is such a low-band gap polymer consisting of an electron-rich cyclopentadithiophene (CPDT) part with alkyl side chains and a benzothiadiazole (BT) electron-deficient part $^{9}$ (structure in Figure 1). The coupling of the CPDT and the BT groups creates a hybridization between the highest and lowest occupied molecular orbitals of the two groups, which produces a low band gap $\left(E_{\mathrm{g}}\right)$ of around $1.4 \mathrm{eV}$ and a hole mobility of up to $10^{-2} \mathrm{~cm}^{2} / \mathrm{V}$ s. ${ }^{8}$ Devices of PCPDTBT (and similar polymers) give solar cell efficiencies above 5\% together with PCBM $\left([6,6]\right.$-phenyl- $\mathrm{C}_{61}$-butyric acid methyl ester, structure in Figure $1)$ as an electron acceptor. ${ }^{10,11}$ PCPDTBT has also been used as a hole-conducting material together with $\mathrm{CH}_{3} \mathrm{NH}_{3} \mathrm{PbI}$ perovskites in mixed organic/inorganic hybrid solar cells. ${ }^{12}$

The side chains on the CPDT unit affect the morphology of the $\mathrm{BHJ}:{ }^{13}$ they provide a spacer between polymeric chains, which allows the PCPDTBT network to readily incorporate PCBM. ${ }^{14}$ The use of the $\mathrm{PC}_{71} \mathrm{BM}$ gives the better solar cell

Received: March 13, 2018

Revised: $\quad$ May 20, 2018

Published: May 24, 2018 

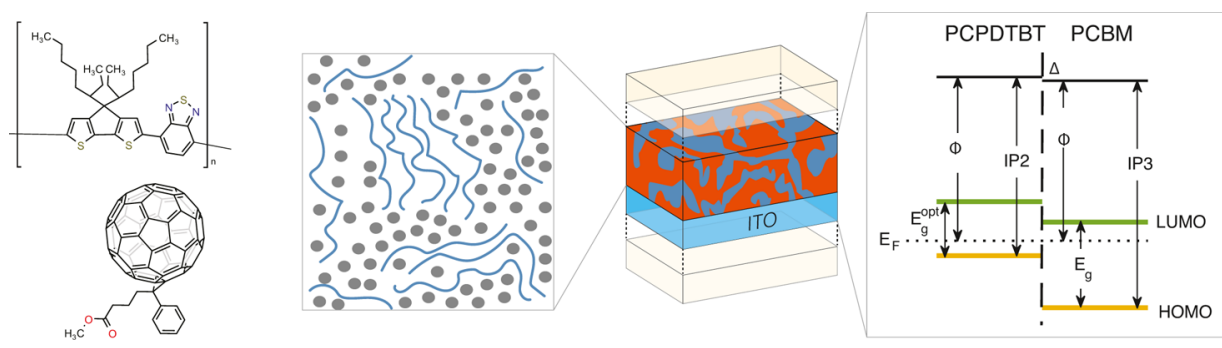

Figure 1. Structures of the PCPDTBT polymer repeat unit and the fullerene derivative PCBM are shown (left); the polymer and PCBM in the thin film form a BHJ on a mesoscopic scale. The studied system is in contact with an ITO substrate. On the right, the energy level diagram between the system constituents are shown, and the labels are discussed in the text.

performance overall. ${ }^{15}$ However, in the present work, the more common and less expensive $\mathrm{PC}_{60} \mathrm{BM}$ molecule has been used.

Ultraviolet photoelectron spectroscopy and X-ray photoelectron spectroscopy (UPS and XPS) have been used to study the valence electronic structure and chemical shifts of the core levels of oxygen, carbon, and sulfur. Recording the kinetic energy $\left(E_{\mathrm{k}}\right)$ of the outcoming photoelectrons from the system at a fixed photon energy $\hbar \omega$ [e.g. $21.2 \mathrm{eV}$ (He I) for UPS and $1486.7 \mathrm{eV}(\mathrm{Al} \mathrm{K} \alpha)$ for XPS], the binding energies $E_{\mathrm{b}}$ of the electronic states in the system can then be deduced via Einstein's relation for the photoelectric effect: $E_{\text {kin }}=\hbar \omega-E_{\mathrm{b}}+$ $\Phi$ if the work function $\Phi$ for the system is known.

Using tunable X-rays from a synchrotron source offers the possibility to study the unoccupied density of states via X-ray absorption in the material. The X-rays may also be tuned to a specific resonance in the system, thus preparing a specific coreexcited state that may decay through autoionization. If we study the electron kinetic energies emanating from the decay of this state, we may call this a resonant Auger spectrum (in analogy with normal Auger processes following a core ionization). ${ }^{16} \mathrm{X}$ ray absorption spectroscopy (XAS) and resonant Auger spectroscopy (RAS) have been used on polymers to investigate how conjugation length, $\pi$-stacking, and various morphological properties affect the electronic structure. ${ }^{17-20}$

Photogeneration and charge separation in organic optoelectronic materials have been recently reviewed. ${ }^{3}$ In Figure 2, charge separation at the interface between PCPDTBT and PCBM is shown. The left side shows photogenerated charge transfer (CT) upon optical excitation. A simple model for this process is Marcus theory, ${ }^{21}$ described later in detail.

In this paper, the CT dynamics from a core-excited state of PCPDTBT have been studied with the core-hole clock method $^{22,23}$ applied to electron spectra containing kinetic energies in the vicinity of the $S \mathrm{KL}_{2,3} \mathrm{~L}_{2,3}$ normal Auger transition with the X-ray photon energy varied over the $\mathrm{S} \mathrm{K}$ edge resonance. This method has been successfully applied to systems similar to the one studied, for example, polythiophenes, alkanethiolates, P3HT:PCBM blends, and other systems. ${ }^{19,24-27}$ In brief, the method measures the prevalence of electrons that emanate from a coherently excited process (i.e., a scattering process where the energy of the ejected electron reflects the excitation energy) relative to electrons that originate from a process that lacks the said coherence. ${ }^{28-30}$ Depending on the lifetime of the excited core hole, processes occurring on the femtosecond and attosecond timescales can thus be studied. $^{31,32}$

To our knowledge, the PCPDTBT:PCBM system has only been studied once prior with RAS and then at excitation energies around the nitrogen K-edge. ${ }^{33}$ No changes in those

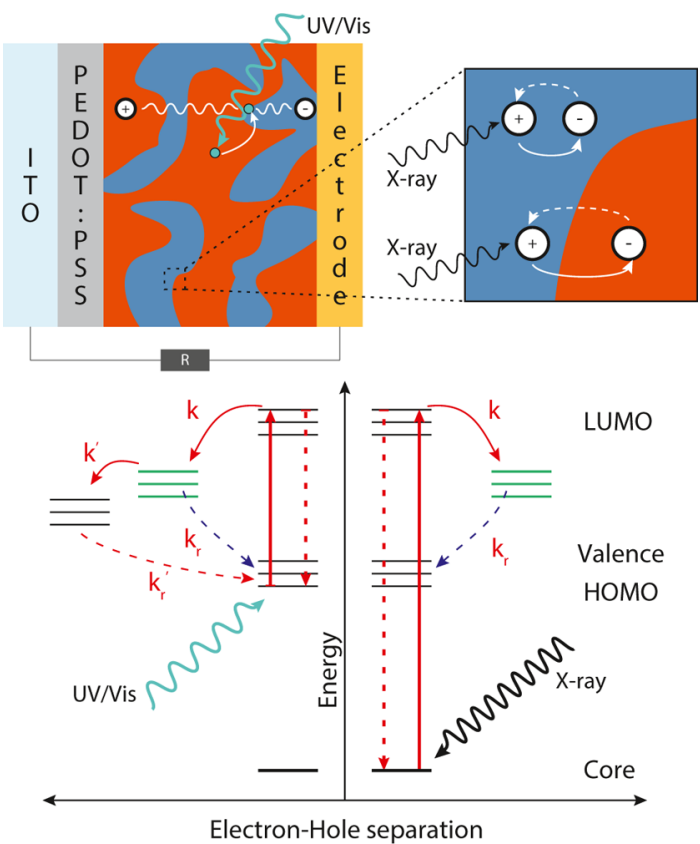

Figure 2. Charge separation in an organic solar cell (ITO/ PEDOT:PSS and a metal electrode with a polymer:PCBM blend in between) using UV/vis or X-ray photons. UV/vis light creates excitons that may be separated at an interface (top, left). X-rays excite with chemical selectivity parts of the system. This enables the study of processes that are either intramolecular or occur at interfaces.

spectra were observed upon changing the morphology of the studied system.

\section{MATERIALS AND METHODS}

Sample Preparation. Pure PCPDTBT (Belectric OPV, $M_{\mathrm{w}}$ $=56000 \mathrm{~g} \mathrm{~mol}^{-1}$ ) polymers and PCBM (Solenne, purity $>99.5 \%$ ) molecules were used to make thin films for this study. Indium-doped tin oxide (ITO) on glass $(R=33.3 \Omega / \square)$ was used as substrates. The substrates were cleaned by sequential sonication in acetone and isopropyl alcohol, both for $15 \mathrm{~min}$, and then finally treated for $5 \mathrm{~min}$ in a UV/ozone generator. The pristine polymer and the blends were doctor blade-casted from solution (dichlorobenzene, 1 wt $\%$ solution) onto ITO substrates and subsequently annealed at $80{ }^{\circ} \mathrm{C}$ for $5 \mathrm{~min}$ in a $\mathrm{N}_{2}$ atmosphere (yielding structures as depicted in Figure 1, main text). No further treatment of the samples, prior to introduction into the vacuum system, was done.

X-ray Spectroscopies. UPS (He I, $21.2 \mathrm{eV}$ ) and XPS (Al $\mathrm{K} \alpha=1486.7 \mathrm{eV}$ ) measurements were performed in a multichamber UHV system with a base pressure of $5 \times 10^{-10}$ 
mbar, in order to determine the energy level alignment (ELA). The electron energy analyzer used was an Omicron hemispherical analyzer (EA 125) mounted in a chamber with a helium discharge lamp (Leybold-Heraeus UVS10/35) and a conventional X-ray tube (Omicron DAR 400).

Hard XPS measurements were performed at the HIKE end station $^{34}$ on the KMC-1 beamline ${ }^{35}$ at Helmholtz-ZentrumBerlin (BESSY II). The KMC-1 beamline is a bending magnet beamline with a double crystal monochromator, and the beam is focused onto the sample through a paraboloid glass capillary. The system base pressure at room temperature was in the $10^{-9}$ mbar range. The electron kinetic energies were recorded using a Scienta/Omicron R4000 electron energy analyzer. All spectra were recorded while using the $\mathrm{Si}(111)$ monochromator crystal, utilizing the first or third order of the monochromatized light (first order photon energies up to $5 \mathrm{keV}$ ).

All binding energy scales are calibrated using the well-known binding energy position $(84.00 \mathrm{eV})$ of the $\mathrm{Au} 4 \mathrm{f}_{7 / 2}$ core level of a gold foil on the manipulator.

$\mathrm{X}$-ray absorption spectra were recorded by recording the $\mathrm{X}$ ray emission spectrum from the sample using a Bruker XFlash 4010 detector as a function of incoming X-ray energy. The photon energy scales are calibrated using the first and third orders of the incoming photon energy.

The resonant Auger spectra and the sulfur KLL spectra were fitted with a least-squares procedure, with Voigt functions and a Shirley background to obtain areas and peak positions.

Monte Carlo simulations were performed to evaluate the fit results. For peak areas and positions, this yielded variations below the per cent level. The estimated error bars are larger and indicate $10 \%$ uncertainty in the CT time-this should accommodate error correlation between the estimated areas in the ratio as well as the uncertainty in the tabulated S 1s core level lifetime value used to extract the CT times.

\section{RESULTS}

Energy Level Alignment. Photoelectron spectroscopy is a valuable tool to determine band offsets, for example, the ELA at interfaces. $^{36-38}$ The basic parameters measured for pristine PCPDTBT and PCBM films prepared on ITO (Figures S1 and S2) are summarized in Table S1. Although, the work function of each component is not accessible in a blend system, the valence ELA can be studied via core level shifts, assuming a constant energetic separation between the core level and the highest occupied molecular orbital. ${ }^{39,40}$

XPS and hard X-ray photoelectron spectroscopy (HAXPES) measurements show that interface dipoles are almost absent at the PCBM-PCPDTBT interface (see the Supporting Information for details). The binding energy differences between the $O$ 1s and $S 2 p$ core levels for blends with PCPDTBT:PCBM mixing ratios of $1: 1,1: 2$, and $1: 3$ were 369.6, 369.7, and $369.6 \mathrm{eV}$, respectively (Figure S3). This corresponds to an average interface dipole of about $-0.1 \mathrm{eV}$. Between ITO and the polymer, an interface dipole of $+0.3 \mathrm{eV}$ was calculated using the same technique. ${ }^{41}$ Thus, the transfer of an excited electron in the lowest unoccupied molecular orbital (LUMO) of PCPDTBT to the LUMO of PCBM should be possible for all mixing ratios.

Hard X-ray Photoelectron Spectroscopy. The sample integrity was checked using HAXPES of the $\mathrm{C} 1 \mathrm{~s}$ core level and overview spectra recorded with a photon energy of $2100 \mathrm{eV}$ together with the $S 1$ s spectrum recorded at a $6300 \mathrm{eV}$ photon energy. The shake-up structure on the high-binding energy side of the $\mathrm{C}$ 1s main structure (Figure S6) testaments the increasing amount of PCBM in the blends. ${ }^{42}$

The $S$ 1s spectrum contains two distinct components (Figure S4), as observed for the S $2 p$ photoelectron line. ${ }^{41}$ The high binding energy component at $2477.3 \mathrm{eV}$ belongs to sulfur bound to nitrogen in the BT group, whereas the lower binding energy component $(2475.7 \mathrm{eV})$ belongs to sulfur in the CPDT units. In all cases, the ratios between the areas of the two peaks were close to 2 , as expected from the stoichiometry of the polymer.

We obtained different Lorentzian broadenings $\left(\Gamma_{\mathrm{L}}\right.$, related to the core hole lifetime $\tau=\hbar / \Gamma_{\mathrm{L}}$ ) from curve fitting of the two sulfur components. The widths differ systematically by about $150 \mathrm{meV}$ if a Gaussian instrumental broadening is used from the $\mathrm{Au} 4 \mathrm{f}$ calibration. With this type of fit, a stoichiometric ratio close to 2 was obtained, whereas other fitting approaches gave different stoichiometric ratios (as discussed in the Supporting Information) and a fit with different Lorentzian widths, which seems therefore most reasonable. We obtained core-hole lifetimes of 880 as for CPDT and 730 as for BT from this model. However, to be able to compare our CT time to those of other works, we instead use a tabulated core hole lifetime, $\tau_{1 \mathrm{~s}}$, of sulfur of $1.27 \mathrm{fs}$ in the analysis of the resonant Auger data (see below) ${ }^{43}$

XAS: Sulfur K-Edge. In Figure 3, the X-ray absorption spectra of the sulfur K-edge of the PCPDTBT polymer and

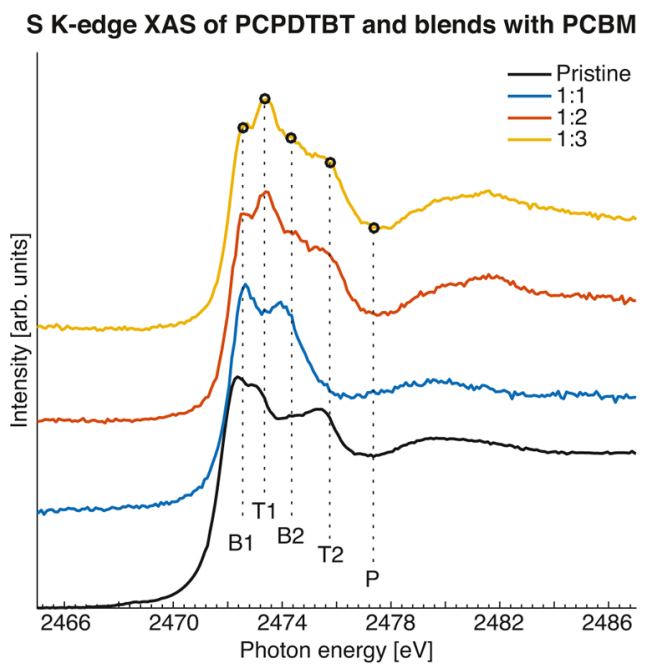

Figure 3. XAS of the pristine polymer and of the different blends. Designations of peaks are discussed in the text.

three blends (1:1, 1:2, and 1:3 weight ratios) with PCBM are shown. The spectra were measured with grazing emission toward the XES detector.

Varying the amount of PCBM in the film changes the X-ray absorption spectra: the relative intensity of the indicated positions at $\mathrm{B} 1$ and $\mathrm{T} 1$ and between $\mathrm{B} 2$ and $\mathrm{T} 2$ changes. The addition of PCBM into a PCPDTBT-like system creates interfaces between the fullerene and the polymer with unoccupied electronic states that are shared between the two. ${ }^{10,44}$ In fact, according to calculations, the three lowest unoccupied orbitals in the combined system coincide with those of $\mathrm{PCBM}^{10}$ that are experimentally determined to reside on the fullerene part of the molecule-as determined by X-ray absorption and resonant photoemission. ${ }^{45}$ 
The PCPDTBT polymer and the 1:1 blend with PCBM were previously studied with X-ray absorption near edge structure. $^{46-48}$ For the benzothiadiazole and the thiophene molecules, K-edge spectra are available in refs 49 and 50. Xray excitation energies denoted by $\mathrm{B}$ arise from excitations primarily associated with the benzothiadiazole parts of the polymer, and energy positions denoted by $\mathrm{T}$ arise from the thiophene parts. ${ }^{46-48}$ The point denoted by $\mathrm{P}$ is slightly below the core ionization threshold.

Earlier investigations ${ }^{46,47}$ found that features around $\mathrm{T} 1$ are mainly polarized in plane, parallel to the polymer backbone; that is, their intensity is at maximum if the electric field vector of the X-rays is parallel to the polymer chain. In contrast, the intensity close to B1 is mainly determined by out-of-plane transitions. In the energy range of B2, in-plane transitions, perpendicular to the backbone, were observed. The variation in intensity between the pure polymer and the different blends can therefore be explained by changes in the preferred molecular orientation. ${ }^{47}$

The changes we observe could then be explained in more detail together with the picture of shared electronic states at the interfaces: (1) the $\pi^{*}$ orbital of the thiophene unit has a large overlap with the $\pi^{*}$ system of PCBM and (2) the $\pi^{*}$ that is more localized to the $\mathrm{S}-\mathrm{N}$ bond of the benzothiadiazole group seems to have less overlap with the fullerene (the B1 and B2 parts of the XAS spectra do not change much with changing blend).

Resonant Auger Spectroscopy. Nonradiative processes that can accompany a core-excited state can be divided into coherent and incoherent ones. ${ }^{29}$ In Figure 4, decay after CT

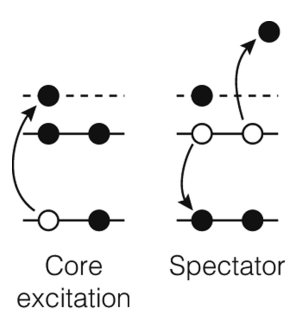

A

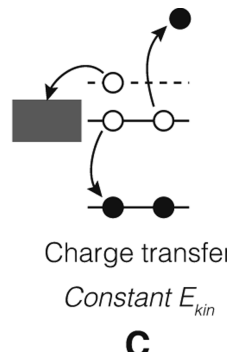

C

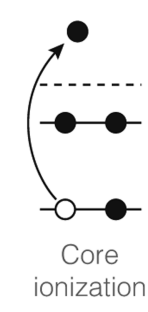

$A^{\prime}$

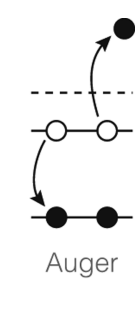

D
Figure 4. X-ray core excited (A) state may decay by the emission of an electron, in a so-called resonant Auger process [in analogy to Auger electron emission (D) upon core ionization $\left(\mathrm{A}^{\prime}\right)$ ]. A spectator decay (B) occurs if the excited electron does not fill the core hole. CT (C), if it occurs during the lifetime of the core hole, yields a final state, which is similar to that of a normal Auger process (D).

(C) and normal Auger decay (D) fall into the noncoherent category-common for all of them is that the energy of the observable emitted electron is not dependent on the excitation energy; this occurs at a constant position on a kinetic energy scale. In the case of the spectator decay (B), the energy is shared between the spectating electron and the emitted electron. This is a coherent process if the spectator electron stays localized (i.e., dispersing or nondispersing on the kinetic energy scale) on the core-excited atom. ${ }^{51}$ Upon CT, energy is shared between the delocalized electron in the solid and the emitted electron. The normal Auger decay occurs after a photoionization, and the energy of the outgoing electron is given by a monopole transition involving the core ionized state and the dicationic final state.
The similarity between the final states of C and D is worth noting and serves as a motivation of trying to observe fingerprints of $\mathrm{CT}$ in the region of kinetic energies belonging to the normal Auger decay. Furthermore, B and C occur only around a resonance, for example, the K-edge, whereas (far) above the resonance, normal photoionization and Auger decays occur.

The dominant transition in the $S$ KLL normal Auger spectrum of PCPDTBT is the $\left({ }^{1} \mathrm{D}_{2}\right)$ transition at electron kinetic energies of $2115 \mathrm{eV}$ accompanied with a second weaker structure at $3 \mathrm{eV}$ higher kinetic energy (Figure S5). The Auger final state is a doubly cationic final state, here with holes in the $S 2 p$ orbitals. In the present system two transitions may be expected since there are two chemically inequivalent sulfur atoms. For the conclusions in this paper, it is enough that we identify the $\left({ }^{1} \mathrm{D}_{2}\right)$ transition as that two-component structure. Henceforth, we will take KLL to refer to this $\mathrm{KL}_{2,3} \mathrm{~L}_{2,3}$ Auger transition. A weaker transition to $\left({ }^{1} S_{0}\right)$ yields Auger electrons of $2105 \mathrm{eV}$ kinetic energy. The measured kinetic energies fall within those measured for other sulfur compounds, where the mean kinetic energy is approximately $2113.9 \mathrm{eV}$ with a standard deviation of $3.8 \mathrm{eV}^{52,53}$

In Figure 5A, a map of the spectra in the electron kinetic energy region of the $S$ KLL normal Auger spectrum for the 1:1 blend is shown. It is recorded with photon energies varying in the vicinity of the S K-edge resonance (hence, if the intensities were integrated along the abscissa, a total electron yield spectrum reminiscent of Figure 3 would result). The electron kinetic energy spectra were recorded with $0.2 \mathrm{eV}$ photon energy increments.

The horizontal lines (B1, T1, B2, T2, and P) indicate which spectra were chosen for detailed least-squares analysis (nomenclature from ref 54). Peaks having a constant kinetic energy (circles and diamonds) and peaks having a constant binding energy (crosses) are indicated. Vertical bars on each line indicate the intensity of that peak in the fit. A small marker indicates that the area of this line is negligible (less than $1 \%$ of the area of the main line).

For photon energies above $2478 \mathrm{eV}$ in Figure 5A, the map is dominated by intensity at the position of the ${ }^{1} \mathrm{D}_{2} S$ KLL. Excitation energies above the ionization threshold for the system open the channel for normal Auger decay. Here, the $\mathrm{S}$ $1 \mathrm{~s}$ binding energy relative to the vacuum level is $2477.4 \mathrm{eV}$, for the 1:2 blend, and the spectral features in Figure $5 \mathrm{G}$ reminisce of the normal Auger spectrum of PCPDTBT taken at a $5 \mathrm{keV}$ photon energy (Figure S5). This is higher than the $S$ 1s photoionization threshold owing to excitations into Rydberg states and also postcollision interaction, which can lead to photoelectron or Auger electron recapture. ${ }^{55-57}$

The resonant Auger spectra taken at points B1, T1, B2, T2, and $\mathrm{P}$ of the $\mathrm{X}$-ray absorption spectra (Figure 3 ) are shown in Figure $5 \mathrm{~B}-\mathrm{G}$ for the $1: 1$ blend. For the other blends, spectra at the corresponding excitation energy points are included in the Supporting Information (Figures S7-S9).

The fits were constructed starting from Figure 5G, which fixes the position of the $\mathrm{CT} /$ normal Auger constant kinetic energy features. Then, using the map (Figure 5A), the spectra in Figure $5 \mathrm{~B}$ were fitted with components corresponding to the intense line at resonance and one at a higher kinetic energy. Again, looking at the map, it can be seen that several lines are needed to fit the spectra in Figure 5C-F. Besides the normal Auger lines, both dispersive and nondispersive spectator lines have been used to fit the spectrum. For polythiophene and 

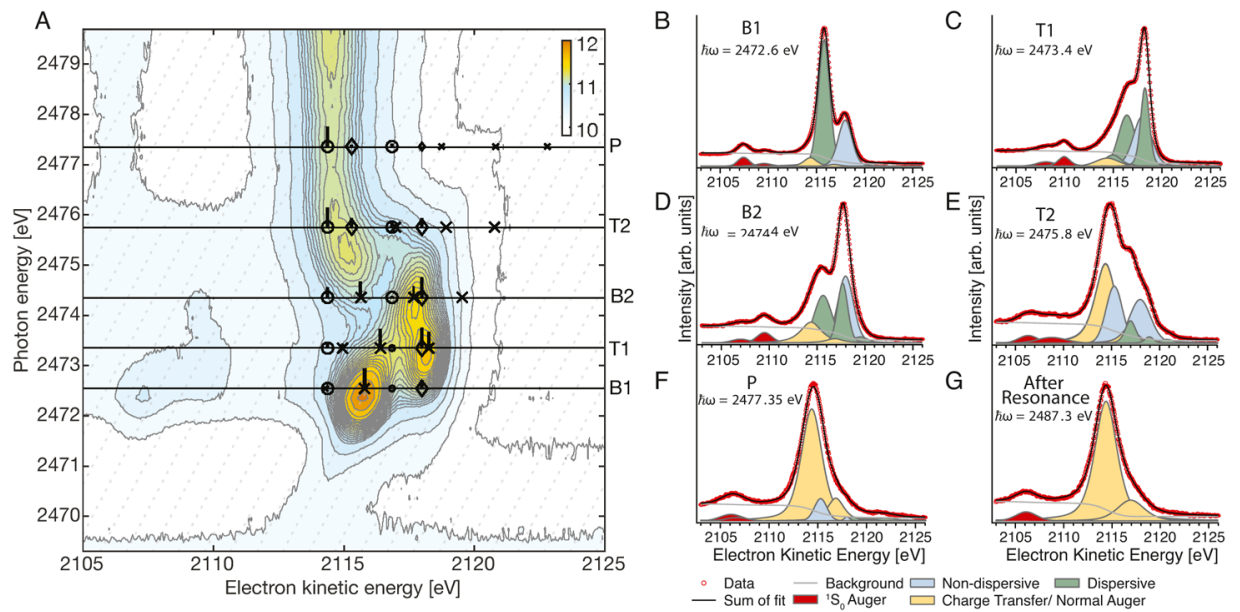

Figure 5. S KLL intensity distribution around the $S$ K-edge for the 1:1 blend is shown in panel (A). The intensity scale is logarithmic, and tilted gridlines indicate constant binding energy (details in the text). Panels $(B-G)$ present the least-squares fit of the spectra at B1 through P.

polybithiophene ${ }^{25}$ and for $\mathrm{PSiF}^{58}$ molecules that contain the same building blocks, these lines are attributed to decays from electrons in $\pi^{*}$ and $\sigma^{*}$ orbitals on the $\mathrm{S}-\mathrm{C}$ and $\mathrm{S}-\mathrm{N}$ bonds or in Rydberg orbitals. It is worth stressing that the map is a key part in finding a way to fit the dataset in a consistent way.

At the B1 transition (Figure 5B), the spectrum is dominated by a dispersing spectator transition slightly above $2115 \mathrm{eV}$ kinetic energy which we can follow in the map moving diagonally with a significant intensity also at points $\mathrm{T} 1$ and $\mathrm{B} 2$. A nondispersing line at $2118 \mathrm{eV}$, present in Figure $5 \mathrm{~B}-\mathrm{F}$, can also be observed along with features coinciding with the energy position of the normal Auger spectrum.

At the $\mathrm{T} 1$ position, new dispersing lines can be observed at 2115 and $2118.3 \mathrm{eV}$, overlapping with the constant kinetic energy lines present at those positions. Spectator lines with constant kinetic energy may come from states where the potential energy surfaces of the intermediate and the final states are parallel. ${ }^{59}$ At the B2 position, no new peaks enter the spectrum. At the T2 position, a new peak at $2115.4 \mathrm{eV}$ appears.

Moving higher up in excitation energy, the resonant contribution to the spectrum becomes more and more faint. At the $\mathrm{P}$ position, most of the intensity is gone and vanished altogether in panel $\mathrm{G}(\hbar \omega=2479 \mathrm{eV})$. Because the Rydberg series converge to the ionization potential, this is expected and indicates the manifold of unoccupied states we excite to have a strong Rydberg character. ${ }^{60}$

Core-Hole Clock. To obtain the CT time $\tau_{\mathrm{ct}}$ we have used eq 1 (see, e.g., Menzel ${ }^{22}$ or Brühwiler et al. ${ }^{23}$ ); the probability of the excited state ending up in a normal Auger like "NA" state or a resonant Auger spectator state either dispersive or nondispersive "RA" during the core hole lifetime is weighed. The CT state is called NA because of the similarity of the final state with respect to the core ionized atom. In the normal Auger case occurring above the ionization threshold, it is dicationic; in the resonant CT state, the core-excited atom becomes dicationic because the spectator electron has tunneled away from the core-excited atom (but it has not left the system, see Figure 4). We use a tabulated core hole lifetime $\tau_{1 \mathrm{~s}}$ of sulfur of $1.27 \mathrm{fs}^{43}$ to make our data directly comparable with work on similar polymer systems. ${ }^{24-26,61}$

In Figure 5, the resonant and normal Auger contributions to the spectra obtained by a least-squares fit are shown. In Figure 6 , we plot the ratios of the resonant intensities, $I_{\mathrm{n}}$, (taken as the

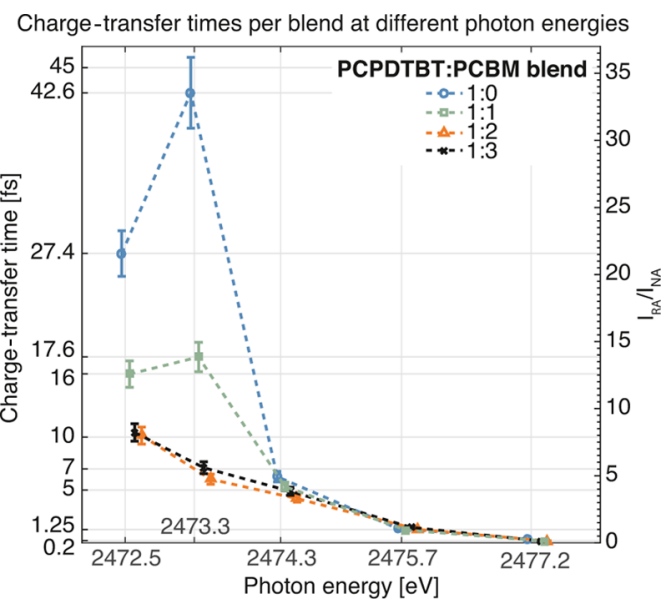

Figure 6. CT times (left ordinate) and ratios between $\mathrm{CT}$ and resonant Auger intensities (right ordinate).

sum of peak areas) and the intensities of the NA channels, $I_{\mathrm{k}}$, and the $\mathrm{CT}$ time calculated from eq 1 versus the photon energy for the different blends.

$$
\tau_{\mathrm{ct}}=\tau_{1 \mathrm{~s}} \cdot \frac{\sum_{\mathrm{RA}} I_{\mathrm{n}}}{\sum_{\mathrm{NA}} I_{\mathrm{k}}}
$$

Two things stand out from Figure 6: (1) The CT time is substantially lowered for the two lowest excitation energies with the addition of PCBM. The largest reduction in time is observed between the pristine polymer and the 1:2 blend (by $86 \%)$. This is a larger difference than those reported for similar systems, for example, $27 \%$ of PSiF-DBT:PCBM. ${ }^{62}$ A $40 \%$ reduction in CT time is reported upon thermal annealing of PSiF-DBT. ${ }^{58}$ (2) The CT times are very similar for all blends for the higher excitation energies. At point $P$, this time is approximately 170 as-roughly half of 360 as measured for the polymer. By adding PCBM, we have therefore doubled the efficiency of CT. Also, the two most dilute blends are similar in all points, whereas for the 1:1 blend, a difference at the lowest two excitation energies persists.

The previously fastest CT time derived using the $\mathrm{S}$ K-edge and the core-hole clock was 220 as for a P3HT:PCBM blend. ${ }^{26}$ We observe CT dynamics occurring from 43 fs down to a mere 
170 as. Moreover, the changes upon addition of PCBM are significant upon excitations at the sulfur sites of the polymer (in contrast to the nitrogen edge). Behaviors for the CPDT and BT groups are not the same, which we explain by differences in the electronic structure at the interface between PCBM and different parts of the polymer.

\section{DISCUSSION}

Comparison of Core-hole Clock Spectroscopy and Marcus Theory. A simple model for CT processes is Marcus theory ${ }^{21}$ where the CT rate depends on the electronic coupling between constituents $H_{\mathrm{DA}}$, the temperature $T$, and the activation energy of the reaction $\Delta G^{*}$, which, in turn, depends on the Gibbs free energy for CT and the reorganization energy $\lambda$ of the system:

$$
k=\left|H_{\mathrm{DA}}\right|^{2} \sqrt{\frac{4 \pi}{h^{2} k_{\mathrm{B}} T \lambda}} \mathrm{e}^{-\left(\Delta G * / k_{\mathrm{B}} T\right)}
$$

with $\Delta G^{*}=\left(\Delta G^{\circ}+\lambda\right)^{2} / 4 \lambda$ relating the energies (for parabolic diabatic potential energy surfaces).

Using optical wavelengths, the created valence excited state (an exciton) is delocalized. The coupling matrix element $H_{\mathrm{DA}}$ couples wave functions of different parts of the system; if a valence bond picture is chosen, off-diagonal elements of the Hamiltonian can be written as $H_{\mathrm{DA}}=A \mathrm{e}^{-\mu\left(r_{\mathrm{D}}-r_{\mathrm{A}}\right)} \cdot{ }^{63}$ The coupling strength depends exponentially on the equilibrium distances $r$ and constants. This is a framework for discussing how charge separation occurs in OPVs at different timescales, as depicted in the top left and bottom in Figure 2 using rates such as $k$ and $k^{\prime}$ : a photoexcitation (femtosecond/picosecond) creates an exciton that may diffuse to an interface where the charges can separate and be transported away (femtoseconds to milliseconds), a process limited by several recombination pathways (dashed lines in Figure 2). ${ }^{3}$ On these timescales, vibrational motion and possible rearrangements affect the electronic overlaps.

An excited state created with X-ray absorption has a (core)hole which is extremely localized (see below). By observing the spectrum of electron kinetic energies from the radiationless decay of this state, we get a complementary view on electron dynamics of the system specific to a certain atomic site-here, at the interface- on a shorter timescale (given by the core hole lifetime). By comparing the pristine polymer to the blends with PCBM, differences in the de-excitation dynamics at the interface between polymer and fullerene can be inferred, that is, how the critical "first jump" of the electron fares at the interface between polymer and PCBM (Figure 2). Core excitation is akin to having a negative charge injected into the unoccupied states of the system locally at an atomic site, and the core-hole clock probes the efficiency of transporting a charge away from this site.

The rate of decay of core-excited processes, accessed at X-ray energies, may be analyzed using Fermi's golden rule (eq 3 ). The expression is similar to that given above:

$$
k=\frac{2 \pi}{\hbar} \sum_{\mathrm{f}}\left|\left\langle\phi_{\mathrm{f}}|\mathbf{Q}| \phi_{\mathrm{i}}\right\rangle\right|^{2} \rho(E)
$$

but the transition rate $k$ between initial $\left|\phi_{\mathrm{i}}\right\rangle$ and final $\left|\phi_{\mathrm{f}}\right\rangle$ states is coupled via the operator $\mathbf{Q}$ weighted by the density of states at a certain energy. Also, instead of the electronic overlap given by $H_{\mathrm{DA}}$ above for a neutral system, here, we need to describe the decay from a core-excited $N$-electron system into a $N-1$ electron system with an electron in the continuum with a Coulomb matrix element:

$$
\left\langle\phi_{1} \phi_{2}\left|e^{2} / r\right| \phi_{\mathrm{c}} \phi_{\mathrm{e}}\right\rangle\left\langle\psi_{\mathrm{i}}^{N-2} \mid \psi_{\mathrm{f}}^{N-2}\right\rangle
$$

Here, the approximation is made that the other $N-2$ electrons in the system are only screening the electrons 1 and 2 in the final state and the core hole and excited electron in the initial state. The transition between the two states is governed by the Coulomb operator. ${ }^{16}$

What this matrix element tells us, when used in eq 3 , is that the spectrum we observe from a core de-excitation probes the electron density in the vicinity of the core hole because $\phi_{c}$ is strongly localized (in contrast to holes in more diffuse valence orbitals); furthermore, the spectrum contains information about the rate $k$ and the competing processes. Because deexcitation is a monopole transition, it is qualitatively different from direct photoionization, which is a dipole transition. This makes for a quantitative difference in the time-scales studied: 100 s of attoseconds to femtoseconds for monopole transitions (core-hole clock), versus 10s of femtoseconds to picoseconds for UV/vis pump - X-ray probe photoemission spectroscopy. ${ }^{64}$ This makes for a distinctive case for using core-hole clock spectroscopy for this type of studies, not just for the different time regimes but also for the focus on localized effects.

CT in PCPDTBT:PCBM Blends. Owing to the morphology of the composite polymer:PCBM system, we may divide the $\mathrm{CT}$ into two categories: intramolecular; that is, the core-excited electron is conducted away to neighboring parts of the molecule owing to the alternating donor-acceptor structure of the polymer, and intermolecular, where we consider CT to neighboring polymers or PCBM molecules. Adding PCBM to a polymer blend might induce conformational ordering of the polymer chains ${ }^{65}$ and hinder their self-organization ${ }^{48}$ - this affects the orbital overlap for the polymer chains in eq 4 concerning intramolecular CT. However, the intensities in the normal Auger spectra considered here remain unchanged upon PCBM addition (see Figure S5 in the Supporting Information). Our core excitation spectroscopy is a local probe, so locally the morphological changes of the polymer do not seem to change the intramolecular CT rate much.

Calculations on the LUMO states of the blend show that the first unoccupied state is, in fact, predominantly on PCBM. ${ }^{10,44,66}$ If present, these states add new channels for intermolecular CT in eq 3, observable in the resonant Auger spectrum (new channels emerge also in the normal Auger spectrum if there is bonding in the initial state). ${ }^{67}$ Because of the resonant excitation into these states, the observed intensity can be large, even though the overlap is small. For instance, for Ar physisorbed on $\mathrm{Ni}$, the $\mathrm{CT}$ time is $5 \mathrm{fs}^{68}$ The X-ray absorption spectra change with PCBM content for low excitation energies, where the contribution from unoccupied states from PCBM is large according to calculations.

Because X-ray absorption spectra change with PCBM content, we know that the two lowest excitation energies, on the thiophene and on the benzothiadiazole sulfur resonances, overlap with the lowest unoccupied states of PCBM. The available density of states is larger in the blend, and the actual final state is different from that of the pristine polymer.

Adding PCBM dramatically changes the CT time of the coreexcited sulfur. In the core-hole clock model (eq 1), a change in time occurs because the ratio between coherent and incoherent excitations changes (implicitly assuming that the core hole 
lifetime stays constant). Looking at eq 3 with the matrix element in eq 4, one can conclude that a more efficient electron transfer can happen if the overlap in the Coulomb matrix elements changes or that the density of states at the excitation energy in question changes. From the theory cited above, we know that a PCBM addition yields new electronic states in the interface, which can allow intermolecular CT from the coreexcited state. This corresponds to a change in the overlap in eq 4 , adding to the sum in eq 3 .

The CT time becomes shorter with more PCBM in the mixture. By adding more PCBM, we change the density of states at the interface $(\rho(E)$ in eq 3$)$, up to a point when the density of those interfacial states does not increase any more. This may be caused by the blend becoming too dilute to increase the interfacial area between PCBM and PCPDTBT (the mesoscopic structure favoring intermixing ${ }^{14}$ as in Figure $1)$. This is seen as the CT times being equal for all excitation energies; that is, neither the overlap nor the density of states changes.

The similarity between the CT times for the three high excitation energies may be explained by a transition from the intermolecular to an intramolecular CT regime. With excitation energies higher than the core-level binding energy and the band gap of the polymer, states delocalized over the polymer backbone become accessible for the CT, making it efficient, even in the pristine polymer. Adding PCBM makes this CT more efficient as well, but not to the extent seen for the lowenergy excitations where the intermolecular channels are much less efficient for a pristine system.

We observe large changes in the X-ray absorption and resonant Auger spectra upon changing the morphology of the system-this is in contrast to the study done using the nitrogen edge. $^{33}$ The effects we observe are comparably smaller on excitation from the sulfur site on the benzothiadiazole group, which suggests that the degree of localization is stronger on that site. The contrast between the different parts in the S KLL region, as presented in the map in Figure 5, is larger than that for the resonant Auger spectra taken at the nitrogen K-edge, which may have contributed to no spectral changes observed upon changing the morphology.

\section{CONCLUSIONS}

The largest change in CT time for the PCPDTBT:PCBM system compared to the pristine polymer is $86 \%$ (27\% for PSiFDBT:PCBM ${ }^{62}$ ), and it also exhibits the fastest CT time recorded for polymer blends: 170 as $(220$ as being the fastest previously measured time, for a P3HT:PCBM blend ${ }^{26}$ ). The changes in the presently studied system are larger than those in similar systems, suggesting that PCPDTBT:PCBM favors delocalization of the core-excited electrons via intermolecular CT if compared to P3HT and PSiF-DBT.

Adding more PCBM to the presently studied system beyond 1:2 blend does not change the CT time. The decrease in the CT time from the core-excited state going from the pristine polymer to the different blends can be explained from the point of view of Fermi's golden rule: with the observed changes in the unoccupied states of the X-ray absorption (theoretical descriptions of the interface between PCBM and PCPDTBT), the transition rate should increase if additional decay channels are added and also if the density of those states increases. Of course, this works up to a point: for the two most dilute blends, the CT times are the same. The interface between PCBM and the polymer cannot become arbitrarily large because, after a certain mixing ratio, additional PCBM molecules only come into contact with other PCBM molecules and thus do not contribute to the interfacial density of states. This happens somewhere between 1:2 and 1:3 mixing ratios. It is known that this ratio gives the best photovoltaic performance-because we find this ratio with the core-hole clock method (sampling electron dynamics in the low-femtosecond and attosecond regimes), the efficiency of the first jump of the electron is a necessary but not sufficient condition to find a blend with good performance. Even though CT times measured here are very fast, the macroscopic CT can be slower. For instance, in the case of a phthalocyanine-containing molecule, the intermolecular CT time due to strong $\pi-\pi$ interaction is on the order of femtoseconds, whereas the macroscopic CT time is dominated by a hopping mechanism which is much slower. ${ }^{69}$

Although the equations for CT look similar at a glance for $\mathrm{UV} /$ vis and X-ray absorption, Marcus theory and the formulation given for de-excitation of X-ray excited holes differ because the Coulomb matrix element contains a localized core hole, which gives the decay process chemical specificity. The core-hole clock method thus offers insights into where electrons emanating from a specific site, excited into a specific state, want to go and how fast they are getting there.

Hence, we can compare results from the nitrogen edge (no spectral changes upon PCBM addition ${ }^{33}$ ) with information about CT from sulfur in $\mathrm{S}-\mathrm{C}$ and $\mathrm{S}-\mathrm{N}$ environments in PCPDTBT. The core-hole clock method can thus be used to study polymer systems (e.g., find candidates for the best blend) from the point of view of ultrafast electron dynamics from prepared well-defined core-excited state with chemical selectivity. ${ }^{27}$ One can thus obtain information on (shorter) timescales complementary to photoemission from valence excitons excited with optical wavelengths using pump-probe photoelectron spectroscopy that lacks chemical specificity and probes slower processes.

\section{ASSOCIATED CONTENT}

\section{Supporting Information}

The Supporting Information is available free of charge on the ACS Publications website at DOI: 10.1021/acs.jpcc.8b02453.

UPS, XPS of blends including determination of the ELA parameters, HAXPES of $S 1$ s, overview and $C 1$ s core level spectra and S KLL normal Auger electron spectra, and resonant Auger spectra of pristine, 1:1, and 1:3 blends (PDF)

\section{AUTHOR INFORMATION}

\section{Corresponding Author}

*E-mail: andreas.lindblad@physics.uu.se. ORCID ${ }^{\circ}$

F. O. L. Johansson: 0000-0002-6471-1093

U. B. Cappel: 0000-0002-9432-3112

H. Peisert: 0000-0002-9742-5800

A. Lindblad: 0000-0002-9188-9604

Present Address

${ }^{\S}$ Division of Applied Physical Chemistry, Department of Chemistry, KTH Royal Institute of Technology, SE-100 44 Stockholm, Sweden (U.B.C.).

\section{Notes}

The authors declare no competing financial interest. 


\section{ACKNOWLEDGMENTS}

A.L. acknowledges the support from the Swedish Research Council (grant no. 2014-6463) and Marie Sklodowska Curie Actions (Cofund, Project INCA 600398, F.O.L.J. acknowledges financial support from K G Westman's foundation. We thank HZB for the allocation of synchrotron radiation beamtime and Roberto Felix Duarte for assistance at the KMC-1 beamline. We also want to thank the Matariki Network (UppsalaTübingen) for financial support.

\section{REFERENCES}

(1) Brabec, C. J.; Gowrisanker, S.; Halls, J. J. M.; Laird, D.; Jia, S.; Williams, S. P. Polymer-Fullerene Bulk-Heterojunction Solar Cells. Adv. Mater. 2010, 22, 3839-3856.

(2) Benten, H.; Mori, D.; Ohkita, H.; Ito, S. Recent Research Progress of Polymer Donor/polymer Acceptor Blend Solar Cells. J. Mater. Chem. A 2016, 4, 5340-5365.

(3) Ostroverkhova, O. Organic Optoelectronic Materials: Mechanisms and Applications. Chem. Rev. 2016, 116, 13279-13412.

(4) Gevorgyan, S. A.; Heckler, I. M.; Bundgaard, E.; Corazza, M.; Hösel, M.; Søndergaard, R. R.; dos Reis Benatto, G. A.; Jørgensen, M.; Krebs, F. C. Improving, Characterizing and Predicting the Lifetime of Organic Photovoltaics. J. Phys. D: Appl. Phys. 2017, 50, 103001.

(5) Laquai, F.; Andrienko, D.; Deibel, C.; Neher, D. In Elementary Processes in Organic Photovoltaics; Leo, K., Ed.; Springer International Publishing, 2017; pp 267-291.

(6) Liu, C.; Wang, K.; Gong, X.; Heeger, A. J. Low Bandgap Semiconducting Polymers for Polymeric Photovoltaics. Chem. Soc. Rev. 2016, 45, 4825-4846.

(7) Zhu, Z.; Waller, D.; Gaudiana, R.; Morana, M.; Mühlbacher, D.; Scharber, M.; Brabec, C. Panchromatic Conjugated Polymers Containing Alternating Donor/Acceptor Units for Photovoltaic Applications. Macromol. 2007, 40, 1981-1986.

(8) Cheng, Y.-J.; Yang, S.-H.; Hsu, C.-S. Synthesis of Conjugated Polymers for Organic Solar Cell Applications. Chem. Rev. 2009, 109, 5868-5923.

(9) Mühlbacher, D.; Scharber, M.; Morana, M.; Zhu, Z.; Waller, D.; Gaudiana, R.; Brabec, C. High Photovoltaic Performance of a LowBandgap Polymer. Adv. Mater. 2006, 18, 2884-2889.

(10) Van den Brande, N.; Van Lier, G.; Da Pieve, F.; Van Assche, G.; Van Mele, B.; De Proft, F.; Geerlings, P. A Time Dependent DFT Study of the Efficiency of Polymers for Organic Photovoltaics at the Interface with PCBM. RSC Adv. 2014, 4, 52658-52667.

(11) Peet, J.; Kim, J. Y.; Coates, N. E.; Ma, W. L.; Moses, D.; Heeger, A. J.; Bazan, G. C. Efficiency Enhancement in Low-Bandgap Polymer Solar Cells by Processing with Alkane Dithiols. Nat. Mater. 2007, 6, 497-500.

(12) Heo, J. H.; Im, S. H.; Noh, J. H.; Mandal, T. N.; Lim, C.-S.; Chang, J. A.; Lee, Y. H.; Kim, H.-j.; Sarkar, A.; et al. Efficient Inorganic-Organic Hybrid Heterojunction Solar Cells Containing Perovskite Compound and Polymeric Hole Conductors. Nat. Photonics 2013, 7, 486-491.

(13) Morana, M.; Wegscheider, M.; Bonanni, A.; Kopidakis, N.; Shaheen, S.; Scharber, M.; Zhu, Z.; Waller, D.; Gaudiana, R.; Brabec, C. Bipolar Charge Transport in PCPDTBT-PCBM Bulk-Heterojunctions for Photovoltaic Applications. Adv. Funct. Mater. 2008, 18, $1757-1766$.

(14) Gu, Y.; Wang, C.; Russell, T. P. Multi-Length-Scale Morphologies in PCPDTBT/PCBM Bulk-Heterojunction Solar Cells. Adv. Energy Mater. 2012, 2, 683-690.

(15) Boland, P.; Lee, K.; Namkoong, G. Device Optimization in PCPDTBT:PCBM Plastic Solar Cells. Sol. Energy Mater. Sol. Cells 2010, 94, 915-920.

(16) Applications of Synchrotron Radiation: High-Resolution Studies of Molecules and Molecular Adsorbates on Surfaces; Eberhardt, W., Ed.; Springer Series in Surface Sciences; Springer Berlin Heidelberg, 1994; Chapter 6, pp 203-262.
(17) Schmeißer, D. Valence States of Poly(3-Hexyl-Thiophene) As Probed by Photoelectron Spectra at Resonant Excitation. Synth. Met. 2003, 138, 135-140.

(18) Gallet, J.-J.; Jolly, F.; Rochet, F.; Bournel, F.; Dufour, G.; Avila, P. A.; Sirotti, F.; Torelli, P. Resonant Auger Spectroscopy of Poly(4Hydroxystyrene). J. Electron Spectrosc. Relat. Phenom. 2002, 122, 1125.

(19) Friedlein, R.; Braun, S.; de Jong, M. P.; Osikowicz, W.; Fahlman, M.; Salaneck, W. R. Ultra-Fast Charge Transfer in Organic Electronic Materials and at Hybrid Interfaces Studied Using the Core-Hole Clock Technique. J. Electron Spectrosc. Relat. Phenom. 2011, 183, 101-106.

(20) Friedlein, R.; Sörensen, S. L.; Osikowicz, W.; Rosenqvist, L.; Crispin, A.; Crispin, X.; de Jong, M.; Murphy, C.; Fahlman, M.; Salaneck, W. R. Electronic Structure of Conjugated Polymers and Interfaces in Polymer-Based Electronics. Synth. Met. 2003, 135, 275277.

(21) Rozzi, C. A.; Troiani, F.; Tavernelli, I. Quantum Modeling of Ultrafast Photoinduced Charge Separation. J. Phys.: Condens. Matter 2018, 30, 013002.

(22) Menzel, D. Ultrafast Charge Transfer at Surfaces Accessed by Core Electron Spectroscopies. Chem. Soc. Rev. 2008, 37, 2212-2223.

(23) Brühwiler, P. A.; Karis, O.; Mårtensson, N. Charge-Transfer Dynamics Studied Using Resonant Core Spectroscopies. Rev. Mod. Phys. 2002, 74, 703-740.

(24) Kao, P.; Neppl, S.; Feulner, P.; Allara, D. L.; Zharnikov, M. Charge Transfer Time in Alkanethiolate Self-Assembled Monolayers Via Resonant Auger Electron Spectroscopy. J. Phys. Chem. C 2010, 114, 13766-13773.

(25) Arantes, C.; Borges, B. G. A. L.; Beck, B.; Araújo, G.; Roman, L. S.; Rocco, M. L. M. Femtosecond Electron Delocalization in Poly(thiophene) Probed by Resonant Auger Spectroscopy. J. Phys. Chem. C 2013, 117, 8208-8213.

(26) Borges, B. G. A. L.; Veiga, A. G.; Tzounis, L.; Laskarakis, A.; Logothetidis, S.; Rocco, M. L. M. Molecular Orientation and Ultrafast Charge Transfer Dynamics Studies on the P3HT:PCBM Blend. J. Phys. Chem. C 2016, 120, 25078-25082.

(27) Wang, L.; Chen, W.; Wee, A. T. S. Charge Transfer Across the Molecule/metal Interface Using the Core Hole Clock Technique. Surf. Sci. Rep. 2008, 63, 465-486.

(28) Karis, O.; Nilsson, A.; Weinelt, M.; Wiell, T.; Puglia, C.; Wassdahl, N.; Mårtensson, N.; Samant, M.; Stöhr, J. One-Step and Two-Step Description of Deexcitation Processes in Weakly Interacting Systems. Phys. Rev. Lett. 1996, 76, 1380-1383.

(29) Mårtensson, N.; Weinelt, M.; Karis, O.; Magnuson, M.; Wassdahl, N.; Nilsson, A.; Stöhr, J.; Samant, M. Coherent and Incoherent Processes in Resonant Photoemission. Appl. Phys. A 1997, $65,159-167$.

(30) Wurth, W. Resonant Auger Raman Effect for Adsorbates. Appl. Phys. A 1997, 65, 155-158.

(31) Björneholm, O.; Nilsson, A.; Sandell, A.; Hernnäs, B.; Mrtensson, N. Determination of Time Scales for Charge-Transfer Screening in Physisorbed Molecules. Phys. Rev. Lett. 1992, 68, 18921895.

(32) Wurth, W. Femtosecond Dynamics of Adsorbate Charge Transfer Processes. Appl. Phys. A 1997, 65, 597-601.

(33) Gliboff, M.; Sulas, D.; Nordlund, D.; deQuilettes, D. W.; Nguyen, P. D.; Seidler, G. T.; Li, X.; Ginger, D. S. Direct Measurement of Acceptor Group Localization on Donor-Acceptor Polymers Using Resonant Auger Spectroscopy. J. Phys. Chem. C 2014, 118, 55705578.

(34) Gorgoi, M.; Svensson, S.; Schäfers, F.; Öhrwall, G.; Mertin, M.; Bressler, P.; Karis, O.; Siegbahn, H.; Sandell, A.; Rensmo, H.; et al. The High Kinetic Energy Photoelectron Spectroscopy Facility at BESSY Progress and First Results. Nucl. Instrum. Methods Phys. Res., Sect. A 2009, 601, 48-53.

(35) Schaefers, F.; Mertin, M.; Gorgoi, M. KMC-1: A High Resolution and High Flux Soft X-Ray Beamline at BESSY. Rev. Sci. Instrum. 2007, 78, 123102. 
(36) Ishii, H.; Sugiyama, K.; Ito, E.; Seki, K. Energy Level Alignment and Interfacial Electronic Structures at Organic/Metal and Organic/ Organic Interfaces. Adv. Mater. 1999, 11, 605-625.

(37) Lindblad, R.; Cappel, U. B.; O’Mahony, F. T. F.; Siegbahn, H.; Johansson, E. M. J.; Haque, S. A.; Rensmo, H. Energy Level Alignment in $\mathrm{TiO}_{2} /$ Metal Sulfide/Polymer Interfaces for Solar Cell Applications. Phys. Chem. Chem. Phys. 2014, 16, 17099-17107.

(38) Helander, M. G.; Greiner, M. T.; Wang, Z. B.; Lu, Z. H. Pitfalls in Measuring Work Function Using Photoelectron Spectroscopy. Appl. Surf. Sci. 2010, 256, 2602-2605.

(39) Aygül, U.; Hintz, H.; Egelhaaf, H.-J.; Distler, A.; Abb, S.; Peisert, H.; Chassé, T. Energy Level Alignment of a P3HT/Fullerene Blend During the Initial Steps of Degradation. J. Phys. Chem. C 2013, 117, 4992-4998.

(40) El-Sayed, A.; Borghetti, P.; Goiri, E.; Rogero, C.; Floreano, L.; Lovat, G.; Mowbray, D. J.; Cabellos, J. L.; Wakayama, Y.; Rubio, A.; et al. Understanding Energy-Level Alignment in Donor-Acceptor/ Metal Interfaces from Core-Level Shifts. ACS Nano 2013, 7, 69146920.

(41) Aygül, U.; Peisert, H.; Frisch, J.; Vollmer, A.; Koch, N.; Chassé, T. Electronic Properties of Interfaces Between PCPDTBT and Prototypical Electrodes Studied by Photoemission Spectroscopy. ChemPhysChem 2011, 12, 2345-2351.

(42) Felicissimo, M. P.; Jarzab, D.; Gorgoi, M.; Forster, M.; Scherf, U.; Scharber, M. C.; Svensson, S.; Rudolf, P.; Loi, M. A. Determination of Vertical Phase Separation in a Polyfluorene Copolymer: Fullerene Derivative Solar Cell Blend by X-Ray Photoelectron Spectroscopy. J. Mater. Chem. 2009, 19, 4899-4901.

(43) Campbell, J. L.; Papp, T. Widths of the Atomic K-N7 Levels. At. Data Nucl. Data Tables 2001, 77, 1-56.

(44) Grancini, G.; Maiuri, M.; Fazzi, D.; Petrozza, A.; Egelhaaf, H.-J.; Brida, D.; Cerullo, G.; Lanzani, G. Hot Exciton Dissociation in Polymer Solar Cells. Nat. Mater. 2013, 12, 29-33.

(45) Richter, M. H.; Friedrich, D.; Schmeißer, D. Valence and Conduction Band States of PCBM As Probed by Photoelectron Spectroscopy at Resonant Excitation. J. Bionanosci. 2012, 2, 59-65.

(46) Batchelor, D. R.; Aygül, U.; Dettinger, U.; Ivanovic, M.; Tournebize, A.; Mangold, S.; Forster, M.; Scherf, U.; Peisert, H.; Chassé, T. Insight into the Orientation of LBG Polymer Films by XANES Experiment and Calculation. Eur. Polym. J. 2016, 81, 686693.

(47) Ivanović, M.; Aygül, M. I. U.; Dettinger, U.; Tournebize, A.; Polek, M.; Batchelor, D.; Mangold, S.; Forster, M.; Scherf, U.; Peisert, H.; Chassé, T. Electronic Structure and Self-Organization Properties of Low Band Gap Polymers: The Effect of the Introduction of Additional Thiophene Moieties. Sol. Energy Mater. Sol. Cells 2016, 157, 286-294. (48) Aygül, U.; Peisert, H.; Batchelor, D.; Dettinger, U.; Ivanovic, M.; Tournebize, A.; Mangold, S.; Förster, M.; Dumsch, I.; Kowalski, S. Molecular Orientation in Polymer/fullerene Blend Films and the Influence of Annealing. Sol. Energy Mater. Sol. Cells 2014, 128, 119125.

(49) Hitchcock, A. P.; DeWitte, R. S.; Van Esbroeck, J. M.; Aebi, P.; Frenc, C. L.; Oakley, R. T.; Westwood, N. P. C. A Valence- and InnerShell Electronic and Photoelectron Spectroscopic Study of the Frontier Orbitals of 2,1,3-Benzothiadiazole, C6H4SN2, 1,3,2,4Benzodithiadiazine, C6H4S2N2, and 1,3,5,2,4-Benzotrithiadiazepine, C6H4S3N2. J. Electron Spectrosc. Relat. Phenom. 1991, 57, 165-187.

(50) Hitchcock, A. P.; Horsley, J. A.; Stöhr, J. Inner Shell Excitation of Thiophene and Thiolane: Gas, Solid, and Monolayer States. J. Chem. Phys. 1986, 85, 4835-4848.

(51) Föhlisch, A.; Feulner, P.; Hennies, F.; Fink, A.; Menzel, D.; Sanchez-Portal, D.; Echenique, P. M.; Wurth, W. Direct Observation of Electron Dynamics in the Attosecond Domain. Nature 2005, 436, 373-376.

(52) Yu, X.-R.; Liu, F.; Wang, Z.-Y.; Chen, Y. Auger Parameters for Sulfur-Containing Compounds Using a Mixed Aluminum-Silver Excitation Source. J. Electron Spectrosc. Relat. Phenom. 1990, 50, 159-166.
(53) Peisert, H.; Chassé, T.; Streubel, P.; Meisel, A.; Szargan, R. Relaxation Energies in XPS and XAES of Solid Sulfur Compounds. J. Electron Spectrosc. Relat. Phenom. 1994, 68, 321-328.

(54) Aygül, U.; Batchelor, D.; Dettinger, U.; Yilmaz, S.; Allard, S.; Scherf, U.; Peisert, H.; Chassé, T. Molecular Orientation in Polymer Films for Organic Solar Cells Studied by NEXAFS. J. Phys. Chem. C 2012, 116, 4870-4874.

(55) Lindblad, A.; Fink, R. F.; Bergersen, H.; Lundwall, M.; Rander, T.; Feifel, R.; Öhrwall, G.; Tchaplyguine, M.; Hergenhahn, U.; Svensson, S.; et al. Postcollision Interaction in Noble Gas Clusters: Observation of Differences in Surface and Bulk Line Shapes. J. Chem. Phys. 2005, 123, 211101.

(56) Lundwall, M.; Lindblad, A.; Öhrwall, G.; Svensson, S.; Björneholm, O. Neighbor-Induced Photoelectron Recapture in Argon Clusters: A Photon-Energy-Dependent Study of Auger Spectra. Phys. Rev. A: At., Mol., Opt. Phys. 2008, 78, 065201.

(57) Piancastelli, M. N.; Guillemin, R.; Marchenko, T.; Journel, L.; Travnikova, O.; Marin, T.; Goldsztejn, G.; de Miranda, B. C.; Ismail, I.; Simon, M. New Achievements on Relaxation Dynamics of Atoms and Molecules Photoexcited in the Tender X-Ray Domain at Synchrotron SOLEIL. J. Phys. B: At., Mol. Opt. Phys. 2017, 50, 042001.

(58) Garcia-Basabe, Y.; Marchiori, C. F. N.; de Moura, C. E. V.; Rocha, A. B.; Roman, L. S.; Rocco, M. L. M. Charge Transfer Dynamics and Molecular Orientation Probed by Core Electron Spectroscopies on Thermal-Annealed Polysilafluorene Derivative: Experimental and Theoretical Approaches. J. Phys. Chem. C 2014, 118, 23863-23873.

(59) Piancastelli, M. N.; Céolin, D.; Travnikova, O.; Bao, Z.; Hoshino, M.; Tanaka, T.; Kato, H.; Tanaka, H.; Harries, J. R.; Tamenori, Y.; et al. A High-Resolution Study of Resonant Auger Decay Processes in N2O After Core Electron Excitation from Terminal Nitrogen, Central Nitrogen and Oxygen Atoms to the $3 \pi$ LUMO. J. Phys. B: At., Mol. Opt. Phys. 2007, 40, 3357.

(60) Deschler, F.; Da Como, E.; Limmer, T.; Tautz, R.; Godde, T.; Bayer, M.; von Hauff, E.; Yilmaz, S.; Allard, S.; Scherf, U.; et al. Reduced Charge Transfer Exciton Recombination in Organic Semiconductor Heterojunctions by Molecular Doping. Phys. Rev. Lett. 2011, 107, 127402.

(61) Garcia-Basabe, Y.; Yamamoto, N. A. D.; Roman, L. S.; Rocco, M. L. M. The Effect of Thermal Annealing on the Charge Transfer Dynamics of a Donor-Acceptor Copolymer and Fullerene: F8T2 and F8T2:PCBM. Phys. Chem. Chem. Phys. 2015, 17, 11244-11251.

(62) Garcia-Basabe, Y.; Marchiori, C. F. N.; Borges, B. G. A. L.; Yamamoto, N. A. D.; Macedo, A. G.; Koehler, M.; Roman, L. S.; Rocco, M. L. M. Electronic Structure, Molecular Orientation, Charge Transfer Dynamics and Solar Cells Performance in Donor/Acceptor Copolymers and Fullerene: Experimental and Theoretical Approaches. J. Appl. Phys. 2014, 115, 134901.

(63) Shurki, A.; Derat, E.; Barrozo, A.; Kamerlin, S. C. L. How Valence Bond Theory Can Help You Understand Your (Bio)Chemical Reaction. Chem. Soc. Rev. 2015, 44, 1037-1052.

(64) Cappel, U. B.; Plogmaker, S.; Terschlüsen, J. A.; Leitner, T.; Johansson, E. M. J.; Edvinsson, T.; Sandell, A.; Karis, O.; Siegbahn, H.; Svensson, S.; et al. Electronic Structure Dynamics in a Low Bandgap Polymer Studied by Time-Resolved Photoelectron Spectroscopy. Phys. Chem. Chem. Phys. 2016, 18, 21921-21929.

(65) Martin, E. J. J.; Bérubé, N.; Provencher, F.; Côté, M.; Silva, C.; Doorn, S. K.; Grey, J. K. Resonance Raman Spectroscopy and Imaging of Push-Pull Conjugated Polymer-Fullerene Blends. J. Mater. Chem. C 2015, 3, 6058-6066.

(66) Leng, C.; Qin, H.; Si, Y.; Zhao, Y. Theoretical Prediction of the Rate Constants for Exciton Dissociation and Charge Recombination to a Triplet State in PCPDTBT with Different Fullerene Derivatives. J. Phys. Chem. C 2014, 118, 1843-1855.

(67) Lindblad, A.; Bergersen, H.; Pokapanich, W.; Tchaplyguine, M.; Öhrwall, G.; Björneholm, O. Charge Delocalization Dynamics of Ammonia in Different Hydrogen Bonding Environments: Free Clusters and in Liquid Water Solution. Phys. Chem. Chem. Phys. 2009, 11, 1758-1764. 
(68) Föhlisch, A.; Menzel, D.; Feulner, P.; Ecker, M.; Weimar, R.; Kostov, K. L.; Tyuliev, G.; Lizzit, S.; Larciprete, R.; Hennies, F.; et al. Energy Dependence of Resonant Charge Transfer from Adsorbates to Metal Substrates. Chem. Phys. 2003, 289, 107-115.

(69) Friedlein, R.; Braun, S.; de Jong, M. P.; Osikowicz, W.; Fahlman, M.; Salaneck, W. R. Ultra-Fast Charge Transfer in Organic Electronic Materials and at Hybrid Interfaces Studied Using the Core-Hole Clock Technique. J. Electron Spectrosc. Relat. Phenom. 2011, 183, 101-106. 